Ambiente, Comportamiento y Sociedad. 2019, 2 (2), 43-65

DOI: https://doi.org/10.51343/racs.v2i2.473

Fecha de recepción: 15 de junio del 2019

Fecha de aceptación: 30 de setiembre del 2019

\title{
Percepción de riesgo, creencias y normas subjetivas de la conducta proambiental en pobladores del sector ladrillero del distrito de San Jerónimo, Cusco, 2019
}

Risk perception, beliefs and subjective norms of pro-environmental behavior in the residents of the brick sector of the San Jerónimo district, Cusco, 2019

Ana Cornejo ${ }^{1,2}$, Daniel Carbajal ${ }^{2}$, Andy Alvarado ${ }^{2}$, Liz Salinas ${ }^{2}$, Rocío León ${ }^{2}$ \& Fredy S. Monge $^{2}$.

${ }^{1}$ Ministerio de Educación. Cusco, Perú.

${ }^{2}$ Centro de Investigación Ambiente, Comportamiento y Sociedad (CIACOMS). Cusco, Perú.

Correspondencia:

Correo electrónico: lilacornejo.3000@gmail.com

Número de celular: 989979201 


\section{Resumen}

La investigación tiene como objetivo estudiar la relación entre percepción de riesgo, creencias y normas subjetivas pro-ambientales en los sectores de producción de ladrillos del distrito de San Jerónimo, Cusco. Se administraron tres instrumentos previamente validados a una muestra de 205 pobladores que viven en una zona de producción de ladrillos. Se implementó un diseño no experimental correlacional y transversal. Los resultados evidenciaron la relación entre percepción de riesgo y creencias pro-ambientales indicando correlación positiva directa de .43 y un valor de $\mathrm{p}<0.000$; la relación entre percepción de riesgo y normas subjetivas indican correlación positiva .33 y un valor $\mathrm{p}<0.001$ y la relación entre las creencias pro-ambientales y normas subjetivas indican correlación positiva significativa .44 y un valor $\mathrm{p}<0.001$. El resultado descriptivo muestra un nivel alto de percepción de riego (63.4\%), del mismo modo las creencias pro-|ambientales (66.3\%); sin embargo, las nomas subjetivas $(56.1 \%)$ tienen un nivel medio. Por último, se observó que los pobladores de las ladrilleras tienen la intención de disminuir conductas que deterioren el medio ambiente, pero este resultado no es reflejado en su conducta.

Palabras Clave: Percepción de riesgo, creencias proambientales, normas subjetivas, comportamiento proambiental. 


\begin{abstract}
The research aims to study the relationship between risk perception, beliefs and subjective pro-environmental norms in the brick production sectors of the San Jerónimo district, Cusco. Three previously validated instruments were applied to a sample of 205 villagers living in a brick production area. A correlational and transversal non-experimental design was implemented. The results showed the relationship between risk perception and proenvironmental beliefs indicating direct positive correlation of .43 and a p-value $<0.000$; the relationship between risk perception and subjective norms indicating positive correlation .33 and a p-value $<0.001$; and the relationship between pro-environmental beliefs and subjective norms indicating significant positive correlation .44 and a p-value $<0.001$. The descriptive result shows a high level of risk perception (63.4\%), similarly the pro-environmental beliefs (66.3\%); however, the subjective norms (56.1\%) have a medium level. Finally, it was observed that the inhabitants of the brick factories have the intention of reducing behaviors that deteriorate the environment, but this result is not reflected in their behavior.
\end{abstract}

Keywords: Risk perception, pro-environmental beliefs, subjective norms, pro-environmental behaviour. 


\section{Introducción}

Los cambios en la naturaleza debido a la cadena de alteraciones en los ecosistemas afectan diversas formas de vida, principalmente a las poblaciones en condiciones de vulnerabilidad (Gonzales et al, 2009). Un elemento determinante para el deterioro del medio ambiente es la conducta humana debido al descontrol y abuso de la naturaleza; generando impactos sobre los elementos ambientales, humanos y sociales (Bolzan, 2008). Por lo tanto, es importante realizar estudios desde un punto de vista psicosocial ambiental para ayudar a variar creencias, normas, actitudes, así como comportamientos en beneficio de la biosfera y del hombre contribuyendo a la disminución del cambio climático.

La contaminación ambiental es generada por el comportamiento humano, debido a la equivocada forma de ver a la naturaleza. Para entender esta problemática es necesario estudiar los procesos mentales que determinan el comportamiento (Slovic, 2010); en esta investigación se estudió tres procesos mentales (Corral, 2019), como es la percepción de riesgo, creencias y normas subjetivas, se seleccionó como zona de estudio, los centros de producción de ladrillos, conocidas como ladrilleras ubicadas en el distrito de San Jerónimo Cusco. Como se sabe es una zona de alta vulnerabilidad debido a los altos niveles de contaminación ambiental, sobre todo la contaminación del aire (Halanocca y Huaman, 2015).

Determinar los niveles de percepción de riesgo en las personas que experimentan estas condiciones que en definitiva afectan su seguridad física y/o psicológica. Además, analizar las creencias pro-ambientales consideradas como una actitud crítica y reflexiva sobre el cuidado del medio ambiente en general, del mismo modo las creencias ambientales forman parte de un sistema de percepciones, significados y valoraciones acerca del medio ambiente, que reflejan una postura del hombre hacia el mundo. El estudio también considera las normas subjetivas que son el reflejo de la influencia social, la cual se define como la percepción de la 
persona sobre las presiones sociales que le son impuestas para realizar o no realizar un determinado comportamiento (Baldi y García, 2006).

Las zonas de Sucso Aucaylle y Picol Orcopujio ubicadas en el sector de las ladrilleras del distrito de San Jerónimo, lugar de la investigación; donde se estudió la percepción de riesgo, creencias y normas subjetivas de la conducta proambiental, debido a los riesgos que se observó durante la producción de ladrillos y tejas. Además, el estudio se propone identificar las correlaciones entre las creencia y normas subjetivas, la conducta pro-ambiental con la percepción de riesgo. Es por ello, que, la presente investigación estudia el grado de la percepción de riesgo incluyendo peligros potenciales; también, estudia la conducta proambiental en un contexto específico relacionando la variable antes mencionada con la creencias y normas subjetivas; asimismo, las descripciones de las variables en estudio.

\section{Metodología}

La investigación tuvo un enfoque cuantitativo, diseño no experimental, tipo descriptivo correlacional. La muestra fue de 205 sujetos entre mujeres y varones de 18 a 74 años de edad, dedicados a la fabricación y/o venta de ladrillos y tejas, también personas que residen en la zona sin dedicarse a actividades de fabricación y/o venta de tejas y ladrillos, todos ubicadas en las zonas de Sucso Aucaylle y Picol Orcopujio del distrito de San Jerónimo.

\section{Tabla 1}

Características de la muestra

\begin{tabular}{lcc}
\hline Zona & $\mathrm{f}$ & $\%$ \\
\hline Sucso Aucaylle & 107 & 52.2 \\
Picol Orcopijio & 98 & 47.8 \\
\hline Total & 205 & 100 \\
\hline \multicolumn{1}{c}{ Edad } & $\mathrm{f}$ & $\%$ \\
\hline De 18 a 26 años & 92 & 44.9
\end{tabular}




\begin{tabular}{lcc} 
De 27 a 36 años & 39 & 19 \\
De 37 a 46 años & 44 & 21.5 \\
De 47 a 56 años & 19 & 9.3 \\
De 57 a 74 años & 11 & 5.4 \\
\hline \multicolumn{1}{c}{ Total } & 205 & 100 \\
\hline Sexo & $\mathrm{f}$ & $\%$ \\
\hline Femenino & 85 & 41.5 \\
Masculino & 120 & 58.5 \\
\hline Total & 205 & 100 \\
\hline Grado de instrucción & $\mathrm{F}$ & $\%$ \\
\hline Primaria & 49 & 23,9 \\
Secundaria & 118 & 57,6 \\
Técnico & 21 & 10,2 \\
Superior & 17 & 8,3 \\
\hline Total & 205 & 100,0 \\
\hline & & \\
Producción y/o venta & $\mathrm{f}$ & $\%$ \\
de ladrillos y tejas & & \\
& & \\
Si & 124 & 60.5 \\
No & 81 & 39.5 \\
\hline Total & 205 & 100 \\
\hline
\end{tabular}

\section{Instrumentos}

La investigación tiene como instrumentos un cuestionario y dos escalas con escala Likert, es decir, que solo pueden ser contestadas por una escala como: siempre, casi siempre, algunas veces y nunca.

Cuestionario para el levantamiento de datos sociodemográficos.

Para la medición de datos sociodemográficos se realizó con una ficha ad hoc que profundiza el conocimiento de algunas características de la muestra. Esta incluyó variables sociodemográficas como edad, sexo, grado de instrucción, si se dedica o no la producción de ladrillos y/o tejas y la zona de residencia o trabajo. Se tomó en cuenta estos datos 
sociodemográficos porque las investigaciones realizados por diferentes científicos tuvieron como datos relevantes las características sociodemográficas.

\section{Escala de la percepción de riesgo}

La escala de percepción de riesgo asumida por la investigación contiene 8 ítems que describen la amenaza que interpretan las personas ante los cambios climáticos, esta escala parte de la Psicología Ambiental, como un nuevo modelo de percepción de riesgo sobre cambio climático, la preocupación por la contaminación ambiental y el riesgo a la que la actividad humana expone a la población en general, esta, conlleva a tomar acciones anti ambientales o pro ambientales (van der Linden, 2015). Para el análisis se utilizó tres índices, uno global y social con índice alfa de Cronbach de .95 , de riesgo personal con índice .87 y la ultima el de riesgo holístico con índice .96 (van der Linden, 2015). Para el presente estudio utilizaremos la versión adaptada a población peruana de Monge, Brügger y Tobias (2016), que tiene dos dimensiones social y personal.

\section{Escala de creencias proambientales.}

Esta escala mide creencias ambientales, posee 26 ítems sobre temas ambientales específicos relacionados con características naturales y problemas ambientales, y sobre la relación entre el hombre y el medio ambiente (Bolzan, 2008), para la investigación se usa la versión adaptada de Bolzan (2008) esta escala se divide en dos dimensiones: creencias antropocéntricas (10 ítems; $\alpha=.72 ; \mathrm{M}=2.19 ; \mathrm{DS}=.63$ ) y creencias Ecocéntricas (16 ítems; $\alpha=.81 ; \mathrm{M}=4.31 ; \mathrm{DS}=.45) ;$ mide el grado de concordancia con las afirmaciones, a través de una escala Likert de 5 puntos $(1=$ desacuerdo total, $5=$ total acuerdo $)$. 
Procesamiento y análisis de datos

El procedimiento de análisis se realizó con el programa estadístico SPSS versión 23; se realizó el estudio piloto para medir la confiabilidad de las escalas y cuestionario de la investigación con el índice de consistencia interna Alfa de Cronbach, también se realizó un análisis preliminar de K-S para observar la normalidad de los datos, en este caso particular los datos resultaron no paramétricos. Consecutivamente se realizó la interpretación y análisis de la muestra con la prueba de correlación Rho de Spearman (para datos no paramétricos), para efectuar la demostración de hipótesis y la correlación.

\section{Resultados}

Se presenta a continuación los análisis descriptivos e inferenciales basándose en los resultados numéricos de las variables estudiadas percepción de riesgo, creencias y normas subjetivas proambientales, obteniendo tres niveles de corte bajo, medio y alto.

\section{Tabla 2}

Frecuencias descriptivas de los niveles de Percepción de Riesgo, Creencias Pro-Ambientales y Normas Subjetivas

\begin{tabular}{lllll}
\hline Variable & Bajo & Medio & Alto & Total \\
\hline $\begin{array}{l}\text { Percepción de } \\
\text { riesgo }\end{array}$ & $12.2 \%$ & $24.4 \%$ & $63.4 \%$ & $100 \%$ \\
$\begin{array}{l}\text { Creencias } \\
\text { proambientales }\end{array}$ & $0,00 \%$ & $33.7 \%$ & $66.3 \%$ & $100 \%$ \\
$\begin{array}{l}\text { Normas } \\
\text { subjetivas }\end{array}$ & $11.7 \%$ & $56.1 \%$ & $32.2 \%$ & $100 \%$ \\
\hline
\end{tabular}

De una muestra de 205 personas la tabla 2 presenta que, el $63.4 \%$ de los pobladores de las ladrilleras poseen niveles altos en percepción de riesgo, lo cual perciben al cambio climático como peligroso, además se observa los niveles bajo y medio con el 12.2\%, 24.4\% respectivamente; de acuerdo, a la tabla se puede evidenciar que la mayor parte de los 
pobladores si perciben los niveles de riesgo que se presentan en las ladrilleras ya que son muy notables los cambios que producen afectando su manera de vivir, ocasionando distintos cambios en el clima, flora, fauna y el suelo; los pobladores de las ladrilleras perciben el peligro al que se exponen por su convivencia con la contaminación; también, perciben que las acciones en la producción de ladrillo son parte del cambio climático que es originado por el exceso de gases de efecto invernadero que provocan durante las actividades de producción de tejas y ladrillos. El $66.3 \%$ de la población obtuvo niveles altos en creencias proambientales revelando una postura de ideas a favor del medio ambiente y un $33.7 \%$ nivel medio; por otro lado, los resultados no muestran el nivel bajo de creencias pro ambientes porque en su mayoría los pobladores tiene creencias, ideas a favor del cuidado del medio ambiente, fuera de ser conscientes por todo lo que sucede a su alrededor están a favor de buscar nuevas formas que ayuden a conservar el medio ambiente, la mayor parte de ellos indagan acceder a encontrar nuevas maneras de ayuda ya que el cuidado del medio ambiente también los beneficia a ellos, los pobladores de las zonas ladrilleras creen en la existencia de nuevas modalidades que preserven su ambiente y de esta forma los cambios no los afecten. Con respecto a las normas subjetivas el $56.1 \%$ de la población se encuentra en el nivel medio seguida por el nivel alto con el 32.2\% mostrando que la población estudiada posee tendencia a sentir presión por actuar a favor del medio ambiente y finalmente el nivel bajo con el $11.7 \%$, de acuerdo con estos resultados la mayor parte de los pobladores son conscientes de la realidad que se vive en la zona ladrillera; pero, conciben mayor presión por las actividades que se les puedan pedir las autoridades y cuidados en general para la preservación del medio ambiente y los recursos existentes ya que el temor más grande que ellos tienen es que a causa de las actividades no beneficiosas que realizan para el cuidado del medio ambiente puedan ir en contra de ellos y su trabajo ya que este es el único medio de ingreso que tienen. 


\section{Tabla 3}

Descripción de los niveles de las variables basándonos en las dimensiones

\begin{tabular}{llllll}
\hline Variable & Dimensiones & Bajo & Medio & Alto & Total \\
\hline Percepción de riesgo & Personal & $5.9 \%$ & $26.3 \%$ & $67.8 \%$ & $100 \%$ \\
& Social & $6.3 \%$ & $20.5 \%$ & $73.2 \%$ & $100 \%$ \\
Creencias proambientales & Antropocéntricas & $3.9 \%$ & $68.3 \%$ & $27.8 \%$ & $100 \%$ \\
& Ecocéntrica & $.5 \%$ & $19 \%$ & $80.5 \%$ & $100 \%$ \\
& Cotidianas & $9.3 \%$ & $42.4 \%$ & $48.3 \%$ & $100 \%$ \\
& Normas subjetivas & $100 \%$ & $0.00 \%$ & $0.00 \%$ & $100 \%$ \\
\hline
\end{tabular}

El 67.8\% de la población tiene niveles elevados para valorar los grados de peligrosidad a nivel personal, en tanto el $26.3 \%$ y el $5.9 \%$ en el nivel medio y bajo respectivamente. En la tabla se muestra que la mayor parte de los pobladores son conscientes de los efectos que trae la contaminación de las ladrilleras, afectando de manera directa a la naturaleza; uno de los factores por lo que perciben el nivel de peligrosidad es porque afecta su salud personal y la de su familia, ya que las ladrilleras siempre están en constante actividad y los daños que causan afecta a toda la población que vive alrededores. El $73.2 \%$ de la de los pobladores manifiesta preocupación por el riesgo que atraviesa la sociedad a causa de la contaminación ambiental, $20.5 \%$ en el nivel medio y $6.3 \%$ en el nivel bajo; revelando que los pobladores de la zona ladrillera consideran como peligrosa la contaminación ambiental y por ende la actividad de producción de ladrillo, ya que la sociedad en general ve a las ladrilleras y otras actividades que contaminan la naturaleza. Con respecto a la dimensión ecocéntrica el $80.5 \%$ de la población tiene predisposición para cuidar el medio ambiente contradictorio a su conducta; por otro lado, el nivel medio con el $19 \%$ seguido del nivel bajo representado por el $0.5 \%$ de la población. Se puede evidenciar que la mayor parte de los pobladores que habitan en las zonas ladrilleras muestran interés en encontrar nuevas alternativas, esto quiere decir que buscan soluciones o medios que podrían optarse para tener 
una relación amigable con el medio ambiente; los pobladores de las ladrilleras siendo conscientes del daño que ocasiona la fabricación de tejas y ladrillos tienen ideas de preservar el ambiente evitando usar materiales tóxicos como llantas, plástico entre otros, evitar botar la basura por las calles entre otros aspectos, esto nos indica que las personas tienen un grado de concientización y buscan cuidar el medio que los rodea. Con respecto a la dimensión antropocéntrica el $68.3 \%$ de la población representa el nivel medio, y el $27.8 \%$ de la población representa el nivel alto indicando que la población tiene tendencia a ver el medio ambiente como un instrumento para satisfacer sus necesidades; por otro lado, el $3.9 \%$ de la población se encuentra en el nivel bajo lo que evidencia que la mayor parte de los pobladores de las zonas ladrilleras manifiestan tener conocimiento sobre los beneficios que les ofrece la naturaleza al punto de explotar sus riquezas para poder satisfacer sus necesidades sin precisar las consecuencias que estas traerán consigo y para todo su alrededor. Los pobladores vieron a la naturaleza como el único de sus recursos para tener nuevas estrategias de mejora para ellos mismos sin darse cuenta que acaban con la existencia del medio ambiente con todos los abusos que realizan ya que de manera objetiva primero es cubrir sus necesidades para sobrevivir antes que buscar acciones de desarrollo sostenible. con respecto a la dimensión no cotidiana, el $100 \%$ de la población no se sienten presionados a realizar conductas (denunciar crímenes ambientales, colaborar con organizaciones medio ambientales, entre otros.) en favor del medio ambiente, la mayor parte de ellos no colaboran con organizaciones ambientales que pueden brindar soluciones o nuevas estrategias para el cuidado de la naturaleza que los rodea; uno de los factores principales es que su único medio de ingreso económico es la elaboración de ladrillos; no obstante, son conscientes que estas actividades son un gran factor de contaminación al aire y el medio ambiente por el humo toxico que generan a diario. Con respecto a la dimensión cotidiana el $48.3 \%$ de la población representa el nivel alto seguida por el nivel medio con el $42.4 \%$. En efecto la mayoría de la población 
muestra predisposición para realizar actividades de cuidado al medio ambiente, y muy por debajo se encuentra el nivel bajo con el 9.3\%. También, se pudo observar que la mayor parte de los pobladores están dispuestos a ayudar de otras maneras al cuidado del medio ambiente, ellos optan por realizar pequeños cambios como evitar el uso excesivo de algunos recursos, usar bombillas de bajo consumo, el usar adecuadamente la energía eléctrica apagando todas las maquinarias al momento de dejar de usarlas, evitar arrojar basura, reciclar entre otros, estos actos son como la predisposición más cercana que ellos pueden llegar a realizar para colaborar con el medio ambiente y ello hace que se sientan satisfechos por la ayuda que brindan al cuidado del ambiente.

Por otro lado, para seguir con el procedimiento se analiza la correlación con el estadístico Rho de Spearman entre las tres variables de la investigación, para ello se tendrá el resultado de coeficiente de asociación de Spearman, el cual se encarga de determinar si existe una relación entre las variables.

\section{Tabla 4}

Correlación entre percepción de riesgo, creencias proambientales y normas subjetivas

\begin{tabular}{llclll}
\hline & \multicolumn{2}{c}{ Creencias proambientales } & \multicolumn{2}{l}{ Normas subjetivas } \\
\hline Percepción de riesgo & $\mathrm{r}$ & $.43^{* *}$ & $\mathrm{R}$ & $.33^{* *}$ \\
& $\mathrm{p}$ & 0.000 & $p$ & 0.000 \\
Creencias proambientales & & & $\mathrm{R}$ & $.44^{* *}$ \\
& & & & & 0.000 \\
\hline
\end{tabular}

Se aprecia que la correlación entre las variables de Percepción de riesgo y Creencias proambientales es de $r=.43^{* *}$, este valor representa una relación positiva directa entre estas variables con un valor de $p<0.000$, en este caso se puede evidenciar que la mayor parte de los pobladores de la zona ladrillera tiene conocimiento de los peligros y consecuencias que 
tiene el desgaste y abuso del medio ambiente y la utilización de hornos artesanales debido a que este emite de forma directa gases tóxicos a la atmosfera y entre otros y directamente tienen creencias favorables para ayudar a la preservación de este por otros medio que estén a su alcance como el cuidado del agua, el reciclaje y, otros. Esta es la manera en los que todos ellos realizan su colaboración para el cuidado del medio ambiente; pero, estas creencias no se reflejan en el comportamiento porque la contaminación sigue latente.

Con respecto a la correlación de variables entre Creencias proambientales y normas subjetivas El coeficiente Rho de Spearman es de $r=.44 * *$ lo que indica una relación directa significativa entre estas dos variables, asimismo presenta un valor de $p<.000$. Además se evidencia que la mayor parte de los pobladores de las zonas ladrilleras son conscientes de la existencia de peligro latente al que están expuestos por las actividades que realizan, las cuales no ayudan a la preservación del medio ambiente, generando consecuencias para ellos mismos y no solo por los cambios abruptos que se puedan dar en el medio ambiente si no en el bienestar de ellos mismos, pero tampoco están dispuestos a manifestar todos los cambios que se generan dentro de estas por las actividades que se realizan por el temor de ser perjudicados por las labores que realizan así que prefieren aportar al cuidado del medio ambiente con conductas de cuidado más básicos.

\section{Tabla 5}

\section{Correlación entre dimensiones.}

\begin{tabular}{|c|c|c|c|c|c|c|}
\hline \multicolumn{3}{|c|}{ Rho de Spearman } & \multicolumn{2}{|c|}{ Normas Subjetivas } & \multicolumn{2}{|c|}{ Creencias proambientales } \\
\hline & & & $\begin{array}{c}\text { Dimensión } \\
\text { no } \\
\text { cotidiana }\end{array}$ & $\begin{array}{c}\text { Dimensión } \\
\text { cotidiana }\end{array}$ & $\begin{array}{l}\text { Dimensión } \\
\text { ecocéntrica }\end{array}$ & $\begin{array}{c}\text { Dimensión } \\
\text { antropocéntrica }\end{array}$ \\
\hline \multirow[t]{4}{*}{$\begin{array}{l}\text { Percepción } \\
\text { de riesgo }\end{array}$} & $\begin{array}{l}\text { Dimensión } \\
\text { personal }\end{array}$ & $\begin{array}{l}\text { Coeficiente de } \\
\text { correlación }\end{array}$ & $.25^{* *}$ & $.26^{* *}$ & $.53^{* *}$ & .06 \\
\hline & & Sig. (bilateral) & 0.000 & 0.000 & 0.000 & 0.42 \\
\hline & Dimensión & $\begin{array}{l}\text { Coeficiente de } \\
\text { correlación }\end{array}$ & $.21^{* *}$ & $.28^{* *}$ & $.52^{* *}$ & .11 \\
\hline & social & Sig. (bilateral) & 0.003 & .000 & 0.000 & .135 \\
\hline \multirow[t]{2}{*}{$\begin{array}{l}\text { Normas } \\
\text { Subjetivas }\end{array}$} & $\begin{array}{l}\text { Dimensión } \\
\text { no }\end{array}$ & $\begin{array}{l}\text { Coeficiente de } \\
\text { correlación }\end{array}$ & & & $.34^{* *}$ & $.24^{* *}$ \\
\hline & cotidiana & Sig. (bilateral) & & & 0.000 & 0.001 \\
\hline
\end{tabular}




\begin{tabular}{llcc}
\hline $\begin{array}{l}\text { Dimensión } \\
\text { cotidiana }\end{array}$ & $\begin{array}{l}\text { Coeficiente de } \\
\text { correlación } \\
\text { Sig. (bilateral) }\end{array}$ & $.43^{* *}$ & .13 \\
\hline
\end{tabular}

Esta tabla, muestra la correlación entre las dimensiones de cada variable, en la investigación realizada se obtuvieron los siguientes resultados: en la variable Percepción de riesgo y Creencias proambientales la correlación entre. La dimensión personal y ecocéntricas tiene correlación positiva considerable $(\mathrm{r}=.53 * *)$, además, se tiene un valor de $\mathrm{p}<0.000$; por otro lado, la dimensión social y ecocéntricas presentan una correlación positiva considerable $\left(\mathrm{r}=.52^{* *}\right)$ y un valor de $\mathrm{p}<0.000$. En la variable Normas subjetivas y Creencias proambientales las dimensiones cotidiana y ecocéntrica $\left(\mathrm{r}=.43^{* *}\right)$ y un valor de $\mathrm{p}<0.000, \mathrm{y}$ las dimensiones no cotidiana y ecocéntrica $\left(\mathrm{r}=.34^{* *}\right)$ y un valor de $\mathrm{p}<0.000$ en cada correlación respectivamente, existe una correlación positiva significativa. Por su lado, las correlaciones entre las dimensión personal y antropocéntrica presentan una correlación positiva baja (0.06) siendo la correlación más débil con un nivel de significancia de $\mathrm{p}=0.42$.

\section{Discusión}

El conjunto de variables analizadas en la presente investigación ha sido insuficientemente estudiado en Perú, ciudad de Cusco, debido a la perspectiva del hecho y no la causa; en consecuencia, esta investigación estudia variables de la psicología ambiental que permiten analizar las cogniciones de las personas que viven en condiciones de contaminación ambiental, producto de la quema de ladrillos y tejas como parte de la actividad de los productores de ladrillos.

Los resultados hallados en la investigación nos muestran las relaciones positivas significativas en las variables de estudio, resultados que a su vez son consistentes con otros estudios sobre la relación de la percepción de riesgo (Slovic, 1987; Brügger et al, 2017; Van der Linden, 2015), con las creencias proambientales (González, 2002; Pato et al, 2005; Bechtel et al, 1999; Pato et al, 2006; Aguilar et al, 2006) y las normas subjetivas (Pasquali, 
2014; Fishbein et al,1975; Aguilar et al, 2006; Stern, 2000). Por otro lado, se observó que las correlaciones entre la percepción de riesgo y las normas subjetivas presentan una correlación débil a diferencias de otras investigaciones (Van der Linden, 2015, Corraliza, 2000, Stern, 1992) la diferencia es debido ya que la población de la presente investigación es afectada directamente por la contaminación a diferencia de otros estudios que tiene una población estudiantil y/o urbana. Es necesario precisar que la relación entre las variables es bidireccional mas no una relación causal, es decir que entre las variables de creencias ambientales y normas subjetivas se observa que no existe una relación de dependencia; por lo tanto, las normas subjetivas afectan a las creencias ambientales como estas últimas también pueden afectar a las normas subjetivas.

Las variables estudiadas independientemente, tienen similares resultados a otras investigaciones, en el caso de percepción de riesgo, Corral et al. (2003) halló que la población investigada percibe diferentes tipos de riesgo: riesgo para el ambiente, riesgo para la sociedad y riesgo personal. En muchos de los casos evaluados, un riesgo alto para el ambiente también era percibido como un riesgo mayor para la sociedad y para el individuo. Del mismo modo, la presente investigación tiene semejantes resultados porque las dimensiones social y personal alcanzaron niveles altos con el $73.2 \%$ y $80.5 \%$, la percepción de riesgo en forma general obtuvo un $63.4 \%$ revelando que los pobladores de las ladrilleras (zona de producción de ladrillos) de San Jerónimo perciben que un riesgo alto para el medio ambiente, para la sociedad y ellos mismos.

La investigación realizada por Fajardo (2009), indica que una de las dimensiones de las creencias pro ambientales que más se presenta en la muestra evaluada es la ecocéntrica, y en menor grado la antropocéntrica. Por esta razón, es que la mayoría de los entrevistados indica sobre el medio ambiente, que es de una entidad que está en armonía, regularidad e igualdad con ellos y los seres humanos. También observamos que respecto a la dimensión 
ecocéntrica un $80.5 \%$, considera importante este comportamiento hacia el medio ambiente; en consecuencia, se puede interpretar como la predisposición que tienen los individuos de practicar estas conductas orientadas a favorecer el cuidado del medio ambiente.

En la investigación de Pasquali (2014) en cuanto a las normas subjetiva se observó que un $40,9 \%$ se siente "a veces" obligado a realizar acciones proambientales y un $34,7 \%$ “ocasionalmente" apuntando así a que pareciera que hay un poco de sentido de responsabilidad en relación a las conductas proambientales. En nuestra investigación la dimensión no cotidiana, destaca con un total de $100 \%$ en el nivel bajo, revela que los pobladores de las ladrilleras no se sienten obligados a realizar conductas proambientales no cotidianas; enfocándose en conductas cotidianas. Por ende, las normas subjetivas son la variable que influyen en la toma de decisiones sobre las conductas finales que posibilitaría que los pobladores realizar o no conductas notables a favor del medio ambiente.

Finalmente, también se determinó la importancia de la necesidad del estudio de percepciones de riesgo, como un elemento predictor de los comportamientos pro ambientales, sobre todo en escenarios de mayor vulnerabilidad como son las fábricas de ladrillos. Además, se identificaron comportamientos que pre disponen a los individuos a elegir conductas amigables y de cuidado con el medio ambiente. En este sentido, resulta necesario promover estor comportamientos, que generan mayor conciencia sobre el medio ambiente en las poblaciones expuestas a condiciones que ponen en riesgo su salud y bienestar, como el caso de las ladrilleras. Se recomienda que futuros estudios analicen variables demográficas y variables emocionales, que tener una comprensión más amplia del problema.

\section{Declaración de conflicto de intereses.}

Los autores declaran que el presente manuscrito no presenta ningún tipo de conflicto de intereses. 


\section{Referencias}

Ajzen, I. (1991). The Theory of Planned Behavior. Organizational Behavior and Human Decision Proesses, 179-211.

Pato, C., Ros, M., \& Tamayo, Á. (2005). Creencias y Comportamiento Ecológico: un estudio empírico con estudiantes brasileños. Medio Ambiente y Comportamiento Humano, 522. Retrieved from https://www.researchgate.net/publication/316952978_Creencias_y_Comportamiento_ Ecologico_un_estudio_empirico_con_estudiantes_brasilenos_1

Aguilar Luzón, M. d. (2006). Predicción de la conducta de reciclaje a partir de la teoría de la conducta planificada y desde el modelo del valor, normas y creencias hacia el medio ambiente. Granada: Editorial de la Universidad de Granada .

Aguilar Luzón, M., García Martínez, M., Monteoliva Sánchez, A., \& Salinas Martínez de Lecea, J. (2006). El modelo del valor, las normas y las creencias hacia el medio ambiente en la predicción de la conducta ecológica. Medio Ambiente y Comportamiento Humano, 21-44. Retrieved from https://mach.webs.ull.es/PDFS/Vol7_2/Vol7_2_b.pdf

Ajzen, I. (1991). The Theory of Planned Behavior. Organizational Behavior and Human Decision Proesses, 179-211.

Amatti, L. (2016, 11 21). Nuestro clima. Retrieved from Nuestro clima: http://blog.nuestroclima.com/los-diez-lugares-mas-contaminados-del-mundo/ Aragones y Amerigo. (1998). Psicología ambiental. Madrid: Pirámide.

Baldi López, G., \& García Quiroga, E. (2006). Una aproximación a la psicología ambiental. Fundamentos en Humanidades, VII(13-14), 157-168. 
Barreto y Sandoval. (2014). Análisis longitudinal de los factores determinantes de la efectividad de un programa de comportamiento sustentable (CS) en el desarrollo de patrones de compra, uso y disposición de bienes de consumo y servicios públicos en hogares bogotanos. Bogotá: Fundación Universitaria Konrad Lorenz.

Bolzan, C. (2008). Sistemas de gestión ambiental y comportamiento proambiental de trabajadores fuera de la empresa: Aproximación a una muestra brasileña. Barcelona : Universidad de Barcelona Facultad de Psicología.

Calero, P. P. (2017). Percepción del cambio climatico, ádaptación y estrategias de mitigación de los habitantes de la vereda chorillos de la localidad de suba en Bogotá. Bogotá: Universidad Distrital Francisco José de Caldas.

Castro, R. (2002). ¿Estamos dispuestos a proteger nuestro ambiente? Intención de conducta y comportamiento proambiental. Medio Ambiente y Comportamiento Humano, 107118.

Clark, M. (1995). Changes in Euro-American Values Needed for Sustainability. Journal of Social Issues, 63-82.

Conservación Internacional. (2013). Cómo adaptarse al cambio climático: un manual para la comunidad. Colombia.

Corral Verdugo, V., Frías Armenta , M., \& González Lomelí , D. (2003). Percepción de riesgos, conducta proambiental y variables demográficas en una comunidad de Sonora, México. Región y sociedad, 49-72.

Corral, V. (1996). A structural model of reuse and recycling in Mexico. Environment and Behavior, 665-696.

Corraliza, J. (2000). Estilos de vida, actitudes y comportamientos ambientales. Medio Ambiente y Comportamiento Humano, 31-56. 
Fajardo, D. (2009). Analisis de las creencias ambientales y su relacion con el comportamiento proambiental. Bolivia.

Federación Internacional de los Derechos Humanos. (2013). Informe sobre la situación de La Oroya : cuando la protección de los inversores amenaza los derechos humanos. Lima. Retrieved from https://www.fidh.org/es/region/americas/peru/informe-sobre-lasituacion-de-la-oroya-cuando-la-proteccion-de-los-13239

García, d. C. (2012). Concepto de precepción del riesgo y su repercusión en las adicciones. Health and Addictions / Salud y Drogas, 133-151.

Gifford, R. (2014, 1). Environmental Psychology Matters. Retrieved from Annual Review of Psychology: https://www.annualreviews.org/doi/full/10.1146/annurev-psych-010213115048

Gonzales, J., Earls, J., Araujo, H., Ensor, J., \& Torres , J. (2009). Perspectivas sobre el cambio climático. Tecnología y Sociedad, 135-136.

González, L. A. (2002). La Preocupación por la calidad del medio ambiente. Un modelo cognitivo sobre la conducta ecológica. Madrid: Universidad Complutense de Madrid.

Halanocca y Huamán. (2015, Abril 2). Repositorio de la UNSAAC. Retrieved from http://repositorio.unsaac.edu.pe/handle/UNSAAC/189

Halanocca, Q. Y., \& Huaman, V. R. (2015). Impacto Ambiental generado por el sector ladrillero en el distrito de San Jerónimo - Cusco. cusco: Universidad Nacional de San Antonio Abad del Cusco.

Holahan, J. (1999). Psicología Ambiental. Un enfoque general. México: Limusa.

Holohan, C. J. (2014). Psicología ambiental: Un enfoque general. México : Limusa.

INEI. (2017). XII Censo de Población, VII de Vivienda y III de Comunidades Indígenas. Lima: INEI .

Kantor, J. (1959). Interbehavioral psychology. Chicago: Principia Press. 
Martínez, S. J. (2004). Comportamiento proambiental. Una aproximación al estudio del desarrollo sustentable con énfasis en el comportamiento persona-ambiente. Theomai, 99.

Ministerio del Ambiente. (2010). Guía de Evaluación de Riesgos Ambientales . Retrieved Octubre 11, 2019, from MINAM: http://www.minam.gob.pe/calidadambiental/wpcontent/uploads/sites/22/2013/10/guia_riesgos_ambientales.pdf

Ministerio del Ambiente. (2013-2014). Informe Nacional de Calidad del Aire. Lima. Retrieved from Plataforma digital unica del Estado Peruano : www.minam.gob.pe

Miranda Murillo, L. M. (2013). Cultura ambiental: un estudio desde las dimensiones de valor, creencias, actitudes y comportamientos ambientales. Scielo, 94-105.

Moyano Diaz, E., Cornejo, F., \& Gallardo, I. (2011). CREENCIAS Y CONDUCTAS AMBIENTALES, LIBERALISMO ECONÓMICO Y FELICIDAD. Retrieved from Acta Colombiana De Psicología: https://editorial.ucatolica.edu.co/ojsucatolica/revistas_ucatolica/index.php/actacolombiana-psicologia/article/view/346

Organismo de Evalución y Fiscaliazión Ambiental. (2019, Marzo 15). OEFA presenta resultados de la evaluación ambiental realizada en la zona de influencia de las ladrilleras de San Jerónimo en Cusco. Retrieved from Organismo de Evalución y Fiscalización Ambiental: https://www.oefa.gob.pe/noticias-institucionales/oefapresenta-resultados-de-evaluacion-ambiental-realizada-en-la-zona-de-influencia-delas-ladrilleras-de-san-jeronimo-en-cusco

Palacio, S. A. (2018). Comportamiento Proambiental de una muestra de estuediantes de pregrado de la Universidad de Atoquia. Medellín.

Pasquali, C. (2014). Impacto de la etapa universitaria en la formacion del Comportamiento Proambiental. Caracas: Universidad Central de Venezuela. 
Pastor, R. G. (2000). Conducta interpersonal: ensayo de Psicología Social sistemática. Salamanca: Publicaciones Universidad Pontificia.

Pato, C., \& Tamayo, A. (2006). Valores, Creencias Ambientales y Comportamiento Ecológico de Activismo. Medio ambiente y comportamiento humano, 51-66. Retrieved from https://mach.webs.ull.es/PDFS/Vol7_1/Vol7_1_d.pdf

Peña, G. D. (2017). Creencias y comportamientos proambientales en estudiantes de administración en universidades mexicanas en función del grado de implementación del Sistema de Gestión Ambiental. Barcelona, España.

Rioja, G. d. (2017). Evaluación de impacto ambiental. Retrieved from Evaluación de impacto ambiental: http://www.larioja.org/medio-ambiente/es/prevencion-controlambiental/evaluacion-impacto-ambiental

Rivera Jacinto, M., \& Rodríguez Ulloa, C. (2019, julio 22). Actitudes y comportamientos ambientales en estudiantes de enfermería de una universidad pública del norte del Perú. Retrieved from Revista Peruana de Medicina Experimental y Salud Pública: http://www.scielo.org.pe/scielo.php?script=sci_arttext\&pid=S1726$46342009000300012 \& \operatorname{lng}=\mathrm{es} \& \operatorname{tlng}=\mathrm{es}$.

Roth, E. (2000). Psicología ambiental: interfase entre conducta y naturaleza. Revista Ciencia y Cultura, 63-78.

Ruelas Moscoso, H. (2018, Noviembre 30). Problema de contaminación en el distrito de San Jerónimo continúa y no hay sanción para los responsables. Retrieved from Radio Universal : http://www.radiouniversalcusco.com.pe/noticias/32644-2018-11-29-23$20-56$

Slovic, P. (1987). Perception of risk. Science (New York, N.Y.), 280-285.

Slovic, P. (1987). Percption of Risk. Perception of risk, 280-285.

Slovic, P. (2010). La psicología del riesgo. Scielo, 1-14. 
Stern, P. (1992). Psychological dimensions of global environmental change. Annual Review of Psychology, 43.

Torres Hernández, T., Barreto, I., \& Rincón Vásquez, J. (2015, julio-diciembre). Creencias y normas subjetivas como predictores de intención de comportamiento proambiental. suma psicológica, 86-92. Retrieved from suma psicológica: http://www.redalyc.org/pdf/1342/134242609003.pdf van der Linden, S. (2015). The social-psychological determinants of climate change risk perceptions: Towards a comprehensive model. Journal of Environmental Psychology, $112-124$.

Wesley, S. P. (2002, Enero). Inclusion with Nature: The Psychology Of Human-Nature Relations. Retrieved from Autores y editores: https://www.researchgate.net/publication/279614993_Inclusion_with_Nature_The_Ps ychology_Of_Human-Nature_Relations

Willems, E. (1973). Behavior-Environment Systems: An ecological approach. ManEnvironment Systems. 